\title{
The Bridge Occlusion Balloon for Venous Angioplasty in Superior Vena Cava Occlusion
}

Simon Pecha ${ }^{1}$, MD; Heiko Burger ${ }^{2}$, MD; Liesa Castro ${ }^{1}$, MD; Nils Gosau ${ }^{3}$, MD; Gülsen Atlihan ${ }^{4}$, MD; Stephan Willems ${ }^{3}$, MD; Hermann Reichenspurner ${ }^{1}$, MD; Samer Hakmi ${ }^{1}$, MD

DOI: 10.21470/1678-9741-2018-0289

\begin{abstract}
The Bridge Occlusion Balloon is a compliant balloon, specifically designed for temporary Superior vena cava occlusion in case of Superior Vena Cava laceration during lead extraction procedures.

We here report the first case, using Bridge Occlusion Ballon for a venous angioplasty in a patient with dysfunctional pacemaker leads and symptomatic Superior Vena Cava occlusion. After successful lead extraction, venography was showing a
\end{abstract}

narrow venous canal. Therefore, venous angioplasty using the Bridge balloon was performed.

Especially for high-risk lead extraction cases in patients with Superior Vena Cava stenosis, the Bridge Occlusion Ballon might be used as a combination of a safety-net in case of Superior Vena Cava perforation and for Superior Vena Cava angioplasty.

Keywords: Bridge Occlusion Balloon. Superior Vena Cava. Venous Angioplasty.

\begin{tabular}{ll}
\hline \multicolumn{2}{l}{ Abbreviations, acronyms \& symbols } \\
\hline AF & $=$ Atrial fibrillation \\
AV & $=$ Atrioventricular \\
BOB & $=$ Bridge Occlusion Balloon \\
DDD & $=$ Dual chamber pacemakers \\
ICD & $=$ Implantable cardioverter defibrillator \\
OR & $=$ Oddis ratio \\
RV & $=$ Right ventricle \\
SVC & $=$ Superior vena cava \\
TLE & $=$ Transvenous lead extraction \\
\hline
\end{tabular}

\section{INTRODUCTION}

Venous stenosis or occlusion is common in patients with multiple- and old ICD- or pacemaker leads ${ }^{[1]}$. In patients with symptomatic venous stenosis, a class I recommendation for lead extraction is given ${ }^{[2]}$.

In certain cases, even the extraction of all lead material from vascular space does not result in sufficient vascular recanalization.

'Department of Cardiovascular Surgery, University Heart Center Hamburg, Hamburg, Germany.

2Department of Cardiovascular Surgery, Kerckhoff Clinic Bad Nauheim, Hessen, Germany.

32Department of Electrophysiology, University Heart Center Hamburg, Hamburg, Germany.

${ }^{4}$ Department of Vascular Medicine, University Heart Center Hamburg, Hamburg, Germany.

This study was carried out at the University Heart Center Hamburg, Cardiovascular Surgery, Hamburg, Germany.
Sometimes, the use of an oversized powered extraction tool might help to create a larger canal through the SVC. However, in cases of long-segment venous stenosis, a high risk for recurrent venous occlusion is given, especially when a re-implantation of leads is necessary. In those patients, a venous angioplasty might be helpful, to allow for free drainage of the venous blood through the SVC, thereby resolving symptoms associated with venous stasis $^{[3]}$. The BOB (Spectranetics Corporation, Phillips Medical), is a newly designed, compliant balloon, intended for venous occlusion in case of SVC laceration during TLE ${ }^{[4]}$. However, due to its mechanical properties and specific design for use in the venous system, it might be suitable for venous angioplasty. The device is a compliant, low-pressure balloon. According to its compliance, the risk for venous tear or rupture might be reduced, compared to a rigid high pressure angioplasty balloon. We here report the first case of angioplasty using the $\mathrm{BOB}$ during lead extraction.

\section{CASE REPORT}

An 86-years old male patient was implanted with a rightsided DDD-pacemaker due to Sick sinus syndrome in 2000 and
Corresponding Author:

Simon Pecha

iD https://orcid.org/0000-0002-8747-166X

Department of Cardiovascular Surgery

University Heart Center Hamburg

Martinistr. 52, Hamburg, Germany - Zip Code: 20246

E-mail: s.pecha@uke.de 
received a device exchange and new implantation of a RV lead in 2007.

At the time of lead extraction procedure, the patient presented with a third-degree AV-block and paroxysmal AF. Due to atrial fibrillation, the patient was on phenprocoumon, which was maintained during and after the extraction procedure. The indication for lead extraction was SVC occlusion with symptomatic venous stasis and recurrent dizziness, headaches and jugular swelling (Figure 1).

The patient was treated in a hybrid operating room using general anesthesia with cardiopulmonary bypass circuit standby. The RA and RV leads implanted in 2000 were extracted without difficulties using a $14 \mathrm{Fr}$. $80 \mathrm{~Hz}$ Laser sheath including outer sheaths. Afterwards, a venography was performed, showing only a narrow venous recanalization, carrying a high risk for subsequent re-occlusion.

For this purpose, a super stiff wire has been placed into the 14 Fr. Glide light laser sheath. The wire was then subsequently snared and externalized through the right femoral vein. The wire served as a rail for fluoroscopic guided introduction of the BOB.

The balloon was inflated three times at upper, middle and lower SVC level with subsequent volumes of $40 \mathrm{ml}, 50 \mathrm{ml}$ and 60 $\mathrm{ml}$ of 20/80 contrast agent/saline mixture.

The following venography confirmed an unimpeded drainage of the contrast medium through the SVC to the right atrium (Figure 2).

The patient was extubated in the OR and went back to regular ward. No clinically significant pulmonary embolism or hemodynamic compromise was observed. The further postoperative course was uneventful, the patient had no further jugular swelling or headache and was discharged without any complications. In the 6-month follow-up period, the patient was doing clinically well without signs of jugular swelling, dizziness or headache.

\section{DISCUSSION}

In this case, we have shown for the first time, that the BOB can be successfully used for venous angioplasty.

In case of long-segment venous stenosis, a high risk for recurrence of venous occlusion is given, especially when a reimplantation of leads is necessary. Here, a lead extraction with a powered extraction tool is often not enough, to prevent the patient from recurrent venous stenosis. In those cases, a venous angioplasty might be helpful to resolve the venous stenosis. In some cases, even an angioplasty alone might not be sufficient, especially in the long-term run and some kind of SVC stenting might be necessary ${ }^{[3]}$.

The Bridge occlusion balloon is a newly designed, compliant balloon, that is intended for venous occlusion in cases of SVC laceration during $\operatorname{TLE}^{[4-6]}$. Due to its properties and specific design for the use in the venous system, it might be suitable for venous angioplasty. The device is a compliant, low-pressure balloon and could thereby, minimize the risk for venous tear or rupture, in comparison to a rigid angioplasty balloon. SVC rupture during angioplasty is a rare, but severe complication. Some cases of SVC rupture following angioplasty, have been described in literature, with fatal outcomes, including death. Therefore, the use of a compliant balloon might be favorable to reduce the risk of SVC rupture.

Furthermore, the Bridge occlusion balloon can be prophylactically used in high-risk TLE cases $^{[5,6]}$. Its prophylactic
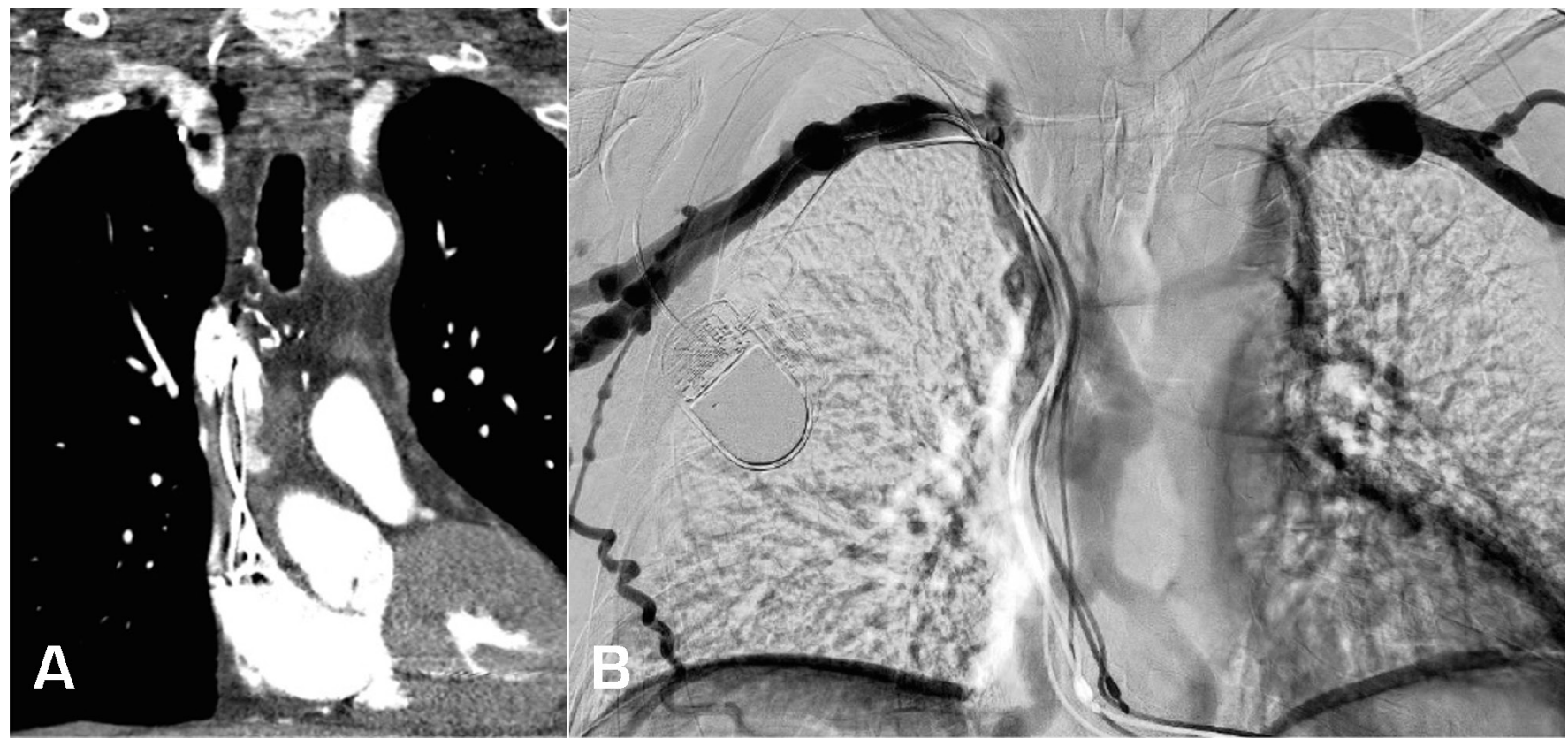

Fig. 1 - A) Preoperative (T Angiography showing SVC occlusion B) Preoperative phlebography showing SVC occlusion. 


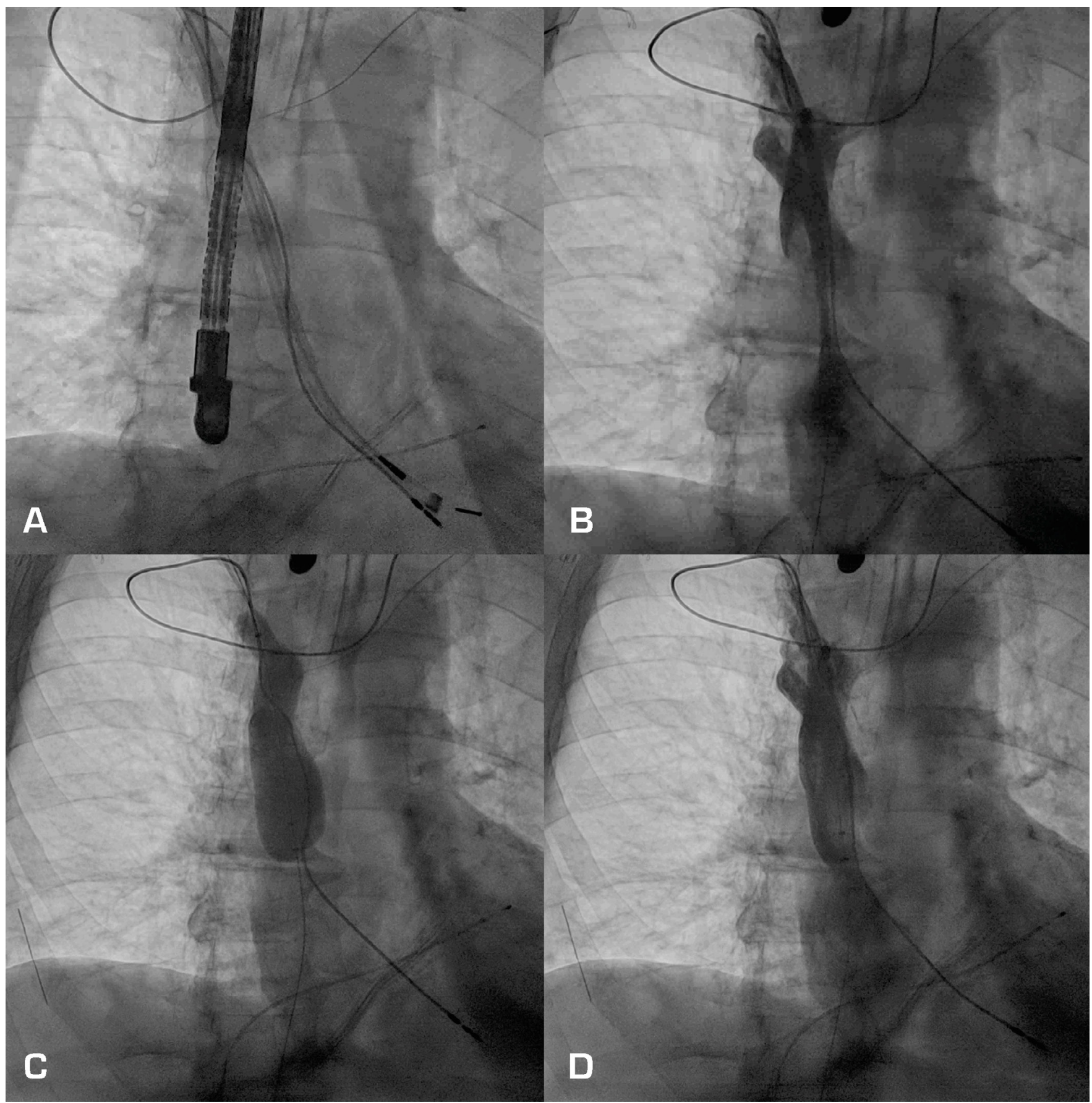

Fig. 2 - A) Laser lead extraction procedure B) Venography after lead extraction showing only a narrow lumen C) Balloon angioplasty using the bridge occlusion balloon D) Venography after balloon angioplasty, showing free contrast agent flow through SVC.

use has been shown to be safe in short and long-term run. Especially, in cases with SVC stenosis, the BOB might be used as a combination of a safety-net in case of SVC perforation and for SVC angioplasty. Although, there is a high rate of subsequent re-stenosis after venous angioplasty reported in literature ${ }^{[3]}$, the patient had no symptoms of venous stasis in the 6-month followup period and was doing clinically well. The fact that the patient was on phenprocoumon due to its atrial fibrillation, might have helped to prevent from re-stenosis. No clear evidence on postoperative anticoagulation after venous angioplasty was found. However, a certain time of anticoagulation treatment seems to be reasonable, to prevent from immediate re-stenosis. Type and timing of anticoagulation needs to be further investigated in larger studies. 


\section{No financial support.}

Conflict of Interest: Samer Hakmi and Heiko Burger are Proctors for Spectranetics (Phillips Medical), Simon Pecha received Speaker honoraria from Spectranetics (Phillips Medical).

\section{Authors' roles \& responsibilities}

SP Conception of the study, manuscript writing; final approval of the version to be published

$\mathrm{HB}$ Revising and approval of the manuscript; final approval of the version to be published

LC Manuscript writing; final approval of the version to be published

NG

Revising and drafting of the manuscript; final approval of the version to be published

GA Acquisition of data, analysis of data and figures; final approval of the version to be published

SW Revising and approval of the manuscript; final approval of the version to be published

HR Revising and approval of the manuscript; final approval of the version to be published

$\mathrm{SH} \quad$ Conception of the study, manuscript writing; final approval of the version to be published

\section{REFERENCES}

1. Wazni O, Epstein LM, Carrillo RG, Love C, Adler SW, Riggio DW, et al. Lead extraction in the contemporary setting: the LExICon Study: an observational retrospective study of consecutive laser lead extractions. J Am Coll Cardiol. 2010;55(6):579-86. doi:10.1016/j.jacc.2009.08.070.

2. Kusumoto FM, Schoenfeld MH, Wilkoff BL, Berul Cl, BirgersdotterGreen UM, Carrillo R, et al. 2017 HRS Expert Consensus Statement on Cardiovascular Implantable Electronic Device Lead Management and Extraction. Heart Rhythm. 2017;14(12):e503-e51. doi:10.1016/j. hrthm.2017.09.001.

3. Brown KT, Getrajdman GI. Balloon dilation of the superior vena cava (SVC) resulting in SVC rupture and pericardial tamponade: a case report and brief review. Cardiovasc Intervent Radiol. 2005;28(3):372-76. doi:10.1007/s00270-004-0001-0.

4. Clancy JF, Carrillo RG, Sotak R, Ram R, Ryu RK, Kennergren C. Percutaneous occlusion balloon as a bridge to surgery in a swine model of superior vena cava perforation. Heart Rhythm. 2016;13(11):2215-20. doi:10.1016/j. hrthm.2016.06.028.

5. Tsang DC, Azarrafiy R, Pecha S, Reichenspurner H, Carrillo RG, Hakmi S. Long-term outcomes of prophylactic placement of an endovascular balloon in the vena cava for high-risk transvenous lead extractions. Heart Rhythm. 2017;14(12):1833-38. doi:10.1016/j.hrthm.2017.08.003.

6. Pecha S, Vogler J, Reichenspurner H, Hakmi S. The bridge occlusion balloon as a safety net in a high-risk transvenous lead extraction procedure. Interact Cardiovasc Thorac Surg. 2018;26(2):360-1. doi:10.1093/icvts/ivx296. 